\title{
Anterior subcutaneous transposition for persistent ulnar neuropathy after neurolysis
}

\author{
*Jort A. N. van Gent, BSc, ${ }^{1}$ Mirjam Datema, MD, ${ }^{2}$ Justus L. Groen, MD, PhD, ${ }^{1}$ \\ Willem Pondaag, MD, PhD, ${ }^{1}$ Job L. A. Eekhof, MD, PhD, ${ }^{3}$ and Martijn J. A. Malessy, MD, PhD ${ }^{1}$ \\ Departments of ${ }^{1}$ Neurosurgery and ${ }^{2}$ Neurology, Leiden University Medical Centre; and ${ }^{3}$ Department of Neurology, Alrijne \\ Hospital, Leiden, The Netherlands
}

\begin{abstract}
OBJECTIVE Little is known about optimal treatment if neurolysis for ulnar nerve entrapment at the elbow fails. The authors evaluated the clinical outcome of patients who underwent anterior subcutaneous transposition after failure of neurolysis of ulnar nerve entrapment (ASTAFNUE).
\end{abstract}

METHODS A consecutive series of patients who underwent ASTAFNUE performed by a single surgeon between 2009 and 2014 was analyzed retrospectively. Preoperative and postoperative complaints in the following 3 clinical modalities were compared: pain and/or tingling, weakness, and numbness. Six-point satisfaction scores were determined on the basis of data from systematic telephonic surveys.

RESULTS Twenty-six patients were included. The median age was 56 years (range 22-79 years). The median duration of complaints before ASTAFNUE was 23 months (range 8-78 months). The median interval between neurolysis and ASTAFNUE was 11 months (range 5-34 months). At presentation, $88 \%$ of the patients were experiencing pain and/ or tingling, $46 \%$ had weakness, and $50 \%$ had numbness of the fourth and fifth fingers. Pain and/or tingling improved in $35 \%$, motor function in $23 \%$, and sensory disturbances in $19 \%$ of all the patients. Improvement in at least 1 of the 3 clinical modalities was found in $58 \%$. However, a deterioration in 1 of the 3 modalities was noted in $46 \%$ of the patients. On the patient-satisfaction scale, $62 \%$ reported a good or excellent outcome. Patients with a good/excellent outcome were a median of 11 years younger than patients with a fair/poor outcome. No other factor was significantly related to satisfaction score.

CONCLUSIONS A majority of the patients were satisfied after ASTAFNUE, even though their symptoms only partly resolved or even deteriorated. Older age is a risk factor for a poor outcome. Other factors that affect outcome might play a role, but they remain unidentified. One of these factors might be earlier surgical intervention. The modest results of ASTAFNUE should be mentioned when counseling patients after failure of neurolysis of ulnar nerve entrapment to manage their expectations. Patients, especially those who are elderly, might even consider not undergoing a secondary procedure. A randomized trial that includes a conservative treatment group and groups undergoing one of the several possible surgical procedures is needed to find the definitive answer for this clinical problem.

https://thejns.org/doi/abs/10.3171/2016.11.FOCUS16451

KEY WORDS ulnar nerve entrapment at the elbow; revision surgery; subcutaneous transposition

$\mathrm{N}$ EUROPATHY of the ulnar nerve at the elbow presents with tingling sensations in the ulnar side of the hand and the fourth and fifth digits, motor weakness, hypesthesia, and pain. The etiology is not always clear, but it can include nerve entrapment at the level of the cubital tunnel. When conservative treatment fails or motor or sensory loss of function is present, surgical treatment can be considered. Neurolysis or transposition are the applied surgical techniques. Based on several meta-analyses, the results of these procedures are approximately equal; however, neurolysis is considered the first choice because of its less invasive character. ${ }^{3,6,8,10,14,17,20}$ This surgical approach leads to a reduction of symptoms in most patients; however, persistent or recurrent symptoms are seen in

ABBREVIATIONS ASTAFNUE = anterior subcutaneous transposition after FNUE; FNUE = failure of neurolysis of ulnar nerve entrapment; UNE = ulnar nerve entrapment SUBMITTED October 30, 2016. ACCEPTED November 29, 2016.

* Mr. van Gent and Dr. Datema contributed equally to this work. 
$10 \%-25 \%$ of the patients. ${ }^{1,10,13}$ These nonresponders form a treatment challenge. Failure of neurolysis of ulnar nerve entrapment (FNUE) is most likely based on 1 of 3 mechanisms: 1) irreversible ulnar nerve damage before surgery, 2) inadequate neurolysis, or 3) new compression of the ulnar nerve at a new site proximal (medial intermuscular septum) or distal (antebrachial fascia) to the medial epicondyle. ${ }^{11,15}$ Revision surgery can be offered if symptoms persist or increase after neurolysis. The results of several retrospective case series of patients who underwent revision surgery have been published; the authors described patients who underwent different primary surgical techniques and different approaches during revision performed by multiple surgeons, which have led to various results. ${ }^{1,2,7,9,15,18}$ The majority of these revision procedures consisted of submuscular transposition. ${ }^{1,2,9,15,18}$ In 1 study, it was clearly stated that subcutaneous transposition was used as revision surgery for FNUE. However, this situation concerned only 4 of 20 patients. The other 16 patients underwent revision surgery for failed submuscular or subcutaneous transposition.? In another study, it was clearly stated that revision surgery for FNUE was performed, but the applied technique was not specified. ${ }^{9}$ Because data in the current literature are limited, definitive conclusions cannot be drawn on the outcome of patients who undergo subcutaneous transposition for FNUE. In our center, subcutaneous transposition is the standard procedure for treating FNUE. To our knowledge, this is the first comprehensive report focused primarily on the results of anterior subcutaneous transposition after FNUE (ASTAFNUE). Our goal was to identify factors associated with outcome and patient satisfaction. We systematically reviewed the outcome of patients who underwent ASTAFNUE performed by an experienced nerve surgeon in a consecutive series of patients treated at our tertiary center for nerve surgery.

\section{Methods \\ Patient Population}

This study was performed with approval of the local medical ethics committee. Each patient provided informed consent. The Leiden University Medical Centre Neurosurgery Department functions as a national tertiary referral center for nerve surgery. We reviewed consecutive patients referred from other hospitals who underwent ASTAFNUE in our center between 2009 and 2014. All subcutaneous transpositions were performed by a single neurosurgeon (M.J.A.M.). FNUE was defined as persistence or an increase of symptoms. We excluded patients who had undergone multiple decompressions before referral or primary transposition of the ulnar nerve. Other exclusion criteria were lesions of the elbow caused by fracture or luxation and ulnar neuropathy caused by prolonged external compression.

\section{Surgical Technique}

The scar over the cubital sulcus that resulted from neurolysis surgery is incised and elongated proximally to the bicipital sulcus and distally to the ulnar ventral side of the lower arm in a gently curving fashion. The subcutis is de- tached from the fascia over the distal part of the medial head of the triceps, the medial epicondyle, and the fascia of the flexor carpi ulnaris muscle, creating a flap. Great care is taken not to damage the branches of the medial antebrachial cutaneous nerve. The ulnar nerve is identified proximal to the scar tissue on top of the medial head of the triceps muscle and behind the distal part of the medial intermuscular septum. Careful stepwise $360^{\circ}$ neurolysis of the ulnar nerve is performed from the proximal to the distal side. The fibrotic scar tissue at the level of the cubital sulcus is thereby resected. The ulnar nerve is transposed ventral to the medial epicondyle. The dorsal plane of the new ulnar trajectory is smoothed in the following 2 steps: 1) by cutting the intermuscular medial septum at the level of the proximal medial epicondyle, and 2) by making a $\mathrm{V}$-shaped incision in the antebrachial fascia to the medial epicondyle but not in the muscles. The transposed position of the ulnar nerve is subsequently secured underneath the subcutaneous flap by 2 sutures that run from the flap to the fascia over the medial epicondyle. The position and adequacy of decompression of the ulnar nerve are tested during passive extension and flexion of the elbow before the wound is closed. Postoperatively, the elbow is immobilized in a sling at $90^{\circ}$ for 48 hours, after which, normal arm movement is encouraged.

\section{Data Collection}

Patient characteristics and preoperative clinical data, categorized into 3 clinical modalities, were collected retrospectively from patient records. These modalities were as follows: 1) pain and/or tingling, 2) weakness/loss of function in the lower arm or hand, and 3) numbness in the ulnar side of the hand. Intraoperative findings were collected from the surgical reports. We noted the presence or absence of subluxation of the ulnar nerve, the presence of fibrosis or adhesions, and the macroscopic aspect, either normal or abnormal, of the ulnar nerve. The ulnar nerve was considered abnormal in cases of a change in the cross-sectional area, with the presence of an intraneural, relatively solid, palpable segment, and/or with an absence of vasa nervorum.

Postoperative data were collected by systematic telephonic surveys. We asked patients about their current complaints in the 3 clinical modalities, and we asked them how they felt about their current symptoms overall compared with their symptoms before the revision surgery, similar to several other studies but more elaborate., ${ }^{2,12}$ Satisfaction was graded on a 6-point scale: 1, complete resolution of symptoms; 2, moderate resolution of symptoms; 3 , no change; 4 , slight increase in symptoms; 5 , moderate increase in symptoms; 6 , severe increase in symptoms. The patients also indicated if, in hindsight, they would opt for revision surgery again, considering their current outcome..$^{2,18}$

\section{Statistical Analysis}

Data analysis was performed using SPSS 20.0. Pearson's product-moment correlation coefficient was calculated for associations of patient symptoms and satisfaction. For statistical analysis, we divided our patients into 2 
groups based on satisfaction score: 1) patients with an excellent or good outcome (complete or partial resolution of symptoms, satisfaction score 1 or 2), and 2) patients who reported a fair or poor outcome (no change in symptoms or deterioration, satisfaction score $3,4,5$, or 6). We used nonparametric tests to compare clinical characteristics between these groups. Sex, occurrence of symptoms before and after surgery, and intraoperative aspect of the nerve were compared using the chi-square test. Age, duration of symptoms, and interval between surgical procedures were compared using the Wilcoxon signed-rank test. Significance was defined as a $\mathrm{p}$ value $<0.05$.

\section{Results \\ Patient Characteristics and Preoperative Findings}

From 2009 until 2014, 33 patients underwent ASTAFNUE. In this period, no patients outside this protocol were treated with submuscular transposition. One patient refused participation, 2 patients had died of an unrelated cause, and 1 patient could not be located. Three patients did not meet the inclusion criteria. The remaining 26 patients were included. The median time to follow-up by telephonic interview was 32 months (range 16-50 months). The median age was 56 years (range 22-79 years). Nine (35\%) patients were female. In 12 patients, surgery was in the right arm, and for 14 patients, the surgery was performed on their dominant arm. The median duration of complaints before ASTAFNUE was 23 months (range 8-78 months). The median interval between neurolysis and ASTAFNUE was 11 months (range 5-34 months). For 3 patients, clinical data at presentation were incomplete. At presentation, $23(88 \%)$ patients experienced pain and/or tingling, 12 (46\%) had weakness, and $13(50 \%)$ had numbness of the fourth and fifth fingers (Table 1).

\section{Intraoperative Findings}

During surgery, the epicondyle-olecranal ligament appeared to be (partially) intact in $24(92 \%)$ patients. Whether the ligament was incompletely cut or "closed" after cutting as a result of the formation of a fibrotic bridge between the cut ends could not be distinguished with certainty. In 13 $(50 \%)$ patients, there was subluxation of the ulnar nerve. In 1 patient, there were clear adhesions. In 15 patients, the ulnar nerve had an abnormal aspect, whereas in 11 patients, the nerve appeared normal. The outcomes between these patients did not differ (Table 2).

\section{Postoperative Findings}

Pain and/or tingling improved in 9 (35\%) patients, motor function improved in $6(23 \%)$, and sensory disturbances improved in 5 (19\%). Improvement in at least 1 of the 3 clinical modalities was found in $15(58 \%)$ patients. Complete resolution of symptoms was achieved in $3(12 \%)$ patients. Fourteen $(54 \%)$ patients reported persistent pain or tingling in the ulnar side of the hand after surgery; this symptom caused difficulties in daily activities in $11(42 \%)$ patients. Eight (31\%) patients reported increased numbness and $13(50 \%)$ patients reported no difference in numbness at the ulnar side of the hand (Table 3).

\section{Patient Satisfaction}

Sixteen $(62 \%)$ patients had an excellent/good outcome after ASTAFNUE, which means that they reported overall either complete $(n=5)$ or partial $(n=11)$ resolution of their symptoms. Ten (38\%) patients had a fair/poor outcome, reporting either no change $(n=5)$ or worsening $(n=5)$ of their symptoms (Table 1). Patient satisfaction was moderately correlated with pain and/or tingling (Pearson correlation coefficient 0.62$)$, more than with weakness $(0.40)$ or numbness $(0.22)$. All the patients with a good outcome would opt for revision surgery again if they had to make the choice; 7 of the 10 patients with a poor outcome regretted their choice for revision surgery in retrospect.

Patients with a fair/poor outcome were 11 years older than patients with an excellent/good outcome (median 62 vs 51 years; $p=0.042$ ). The median duration of symptoms before the neurolysis and time between the FNUE and ASTAFNUE did not differ between patients with an excellent/good outcome and those with a fair/poor outcome. These groups also did not differ in the occurrence of preoperative symptoms or in the appearance of the nerve during ASTAFNUE (Table 2).

\section{Discussion}

To our knowledge, this is the first retrospective case series of patients who underwent ASTAFNUE. The relief of symptoms was modest overall. The most treatable symptom was pain and/or tingling, which was reduced in only $35 \%$ of our patients. However, $62 \%$ of our patients reported an excellent/good outcome on the satisfaction scale, and $73 \%$ would opt for revision surgery again if they had to make the choice. Older age was related to a poor outcome; patients with a fair/poor outcome were, on average, 11 years older than the patients with an excellent/good outcome. In our series, patient satisfaction was relatively good; 16 (62\%) patients reported improvement, and 19 (73\%) patients indicated that, in hindsight, they would opt for revision surgery again. This result is interesting, because only $58 \%$ of the patients experienced improvement in 1 or more of the 3 clinical modalities. Indeed, the correlation of satisfaction with a decrease in symptoms was low. A possible explanation for this contradiction might be the fact that the cessation of progression of symptoms can be perceived as a desirable result, although we assigned patients who reported no change in symptoms to the fair/ poor outcome group.

\section{Outcome of Revision Surgery in the Literature}

A study in 2014 retrospectively compared a group of 28 patients after revision surgery with a control group of patients who had undergone primary surgery. In the revision-surgery group, only 5 of the patients underwent subcutaneous transposition. It is unclear what type of initial surgery was performed in these 5 patients. ${ }^{1}$ Comparable to the patients in our series, the predominant symptoms before revision surgery were pain $(50 \%)$ and paresthesia $(50 \%)$. In this group, the majority $(79 \%)$ of patients who underwent revision surgery reported some degree of symptomatic relief, whereas $53 \%$ of them continued to suffer from constant symptoms, and only $25 \%$ experi- 
TABLE 1. Clinical characteristics, perioperative findings, and outcomes after ASTAFNUE in 26 patients

\begin{tabular}{|c|c|c|c|c|c|c|c|c|c|}
\hline \multirow{2}{*}{$\begin{array}{l}\text { Age } \\
\text { (yrs) }\end{array}$} & \multirow[b]{2}{*}{ Sex } & \multirow[b]{2}{*}{ Side } & \multirow{2}{*}{$\begin{array}{c}\text { Duration of } \\
\text { Complaints (mos) }\end{array}$} & \multirow{2}{*}{$\begin{array}{l}\text { Interval } \\
\text { (mos)† }\end{array}$} & \multirow{2}{*}{$\begin{array}{l}\text { Aspect of } \\
\text { Nerve }\end{array}$} & \multicolumn{3}{|c|}{ Preop/Postop $\ddagger$} & \multirow{2}{*}{$\begin{array}{l}\text { Satisfaction } \\
\text { Score§ }\end{array}$} \\
\hline & & & & & & Pain/Tingling & Motor Function & Sensory Disturbance & \\
\hline 43 & $\mathrm{~F}$ & Rtף & 24 & 6 & Abnormal & $1 / 0$ & $1 / 0$ & $0 / 1$ & 1 \\
\hline 50 & M & $\mathrm{Lt}$ & 36 & 16 & Abnormal & $1 / 0$ & $0 / 1$ & $1 / 1$ & 1 \\
\hline 59 & $\mathrm{~F}$ & $\mathrm{Lt}$ & 36 & 34 & Normal & $1 / 0$ & $1 / 0$ & $0 / 1$ & 1 \\
\hline 79 & $\mathrm{~F}$ & RtT & 14 & 10 & Normal & $1 / 0$ & $0 / 1$ & $1 / 1$ & 1 \\
\hline 22 & $\mathrm{~F}$ & Rtף & $?$ & 8 & Abnormal & $1 / 0$ & $1 / 0$ & $1 / 1$ & 1 \\
\hline 63 & $\mathrm{~F}$ & $\mathrm{Lt} \|$ & 8 & 13 & Abnormal & $1 / 0$ & $0 / 0$ & $0 / 0$ & 2 \\
\hline 42 & M & $\mathrm{Lt}$ & $?$ & 19 & Normal & $1 / 0$ & $0 / 0$ & $0 / 0$ & 2 \\
\hline 48 & $\mathrm{~F}$ & $\mathrm{Lt} ף$ & 8 & 5 & Normal & $0 / 1$ & $1 / 0$ & $1 / 1$ & 2 \\
\hline 52 & $F$ & Lt & 16 & 10 & Normal & $1 / 1$ & $0 / 0$ & $1 / 0$ & 2 \\
\hline 56 & $M$ & Rtף & 19 & 5 & Normal & $1 / 1$ & $1 / 1$ & $0 / 1$ & 2 \\
\hline 62 & $M$ & $\mathrm{Lt}$ & 25 & 12 & Abnormal & $1 / 1$ & $0 / 1$ & $0 / 1$ & 2 \\
\hline 49 & $\mathrm{~F}$ & $\mathrm{Lt}$ & 14 & 12 & Normal & $1 / 1$ & $1 / 0$ & $1 / 0$ & 2 \\
\hline 52 & $\mathrm{~F}$ & Rt $\Uparrow$ & 31 & 12 & Abnormal & $1 / 0$ & $0 / 0$ & $1 / 0$ & 2 \\
\hline 70 & $F$ & $\mathrm{Lt}$ & 8 & 6 & Abnormal & $1 / 0$ & $0 / 0$ & $1 / 1$ & 2 \\
\hline 47 & $M$ & $\mathrm{Lt}$ & 21 & 12 & Normal & $0 / 1$ & $1 / 0$ & $0 / 1$ & 2 \\
\hline 28 & $\mathrm{~F}$ & Rtף & 32 & 23 & Abnormal & $1 / 1$ & $1 / 1$ & $0 / 1$ & 2 \\
\hline 61 & $\mathrm{~F}$ & $\mathrm{Lt}$ & 21 & 7 & Abnormal & $1 / 1$ & $0 / 0$ & $1 / 0$ & 3 \\
\hline 59 & $\mathrm{M}$ & Rt & 8 & 5 & Abnormal & $1 / 1$ & $1 / 1$ & $0 / 0$ & 3 \\
\hline 56 & $\mathrm{~F}$ & Lt & 78 & 18 & Normal & $0 / 1$ & $0 / 1$ & $0 / 1$ & 3 \\
\hline 53 & $F$ & Rt & 49 & 5 & Normal & $1 / 1$ & $1 / 1$ & $0 / 1$ & 3 \\
\hline 54 & $\mathrm{~F}$ & Rt $\Uparrow$ & 32 & 8 & Abnormal & $1 / 1$ & $1 / 1$ & $1 / 1$ & 3 \\
\hline 63 & $\mathrm{~F}$ & $\mathrm{Lt} \|$ & 16 & 6 & Abnormal & $1 / 1$ & $0 / 1$ & $1 / 0$ & 4 \\
\hline 72 & M & $\mathrm{Lt}$ & 23 & 7 & Abnormal & $1 / 1$ & $0 / 1$ & $1 / 1$ & 4 \\
\hline 64 & $M$ & $\mathrm{Rt}$ & 23 & 18 & Normal & $1 / 1$ & $0 / 0$ & $0 / 0$ & 4 \\
\hline 70 & $\mathrm{~F}$ & Rt & $?$ & 18 & Abnormal & $1 / 1$ & $0 / 1$ & $0 / 0$ & 5 \\
\hline 65 & $\mathrm{~F}$ & Rt & 23 & 13 & Abnormal & $1 / 1$ & $1 / 1$ & $1 / 1$ & 6 \\
\hline \multicolumn{10}{|c|}{$\begin{array}{l}\text { ? = unknown. } \\
\text { * Time between first note of complaint until transposition. The ulnar nerve was considered abnormal in cases of change in the cross-sectional area and/or the presence } \\
\text { of an intraneural, relatively solid, palpable segment and/or absence of vasa nervorum. } \\
\text { † Time between neurolysis and ASTAFNUE. } \\
\text { † Symptoms either absent (0) or present (1), before and after anterior subcutaneous transposition. } \\
\S \text { A score of } 1 \text { indicates complete resolution of symptoms; } 2 \text {, moderate resolution of symptoms; } 3 \text {, no change in symptoms; } 4 \text {, slight increase in symptoms; } 5 \text {, moderate } \\
\text { increase in symptoms; and } 6 \text {, severe increase in symptoms. } \\
\text { ๆ Dominant side. }\end{array}$} \\
\hline
\end{tabular}

enced improved symptoms as observed by the clinician. A comparison of the results from this study with our results after ASTAFNUE is not possible. The majority of patients underwent submuscular transposition, and the results are presented for all patients, not subdivided according to type of revision surgery. Another retrospective study described the results of secondary subcutaneous transposition in 20 patients with persistent or recurrent ulnar nerve entrapment (UNE) after a variety of primary procedures. ${ }^{7}$ Outcomes were assessed with a rating system based on symptoms in the same clinical modalities. This study resulted in the improvement of pain in $18(90 \%)$ patients and an excellent/good outcome in 15 (75\%) patients. A comparison of these results to those in our series is not possible, because the vast majority of the cases concerned revision surgery for failed submuscular or subcutaneous transposition. In our study, age was an independent risk factor for poor outcome. A similar effect of age was found in a retrospective study in 30 patients, most of whom underwent submuscular transposition; age older than 50 years was associated with worse outcome. ${ }^{9}$ Therefore, it is very well possible that a study with a younger study group would find somewhat better results than we did. A study in 1991 found revision surgery by submuscular transposition to be highly successful, with relief of pain and paresthesia in all 14 described patients; however, the recovery of motor function and return of sensation were variable, and with an average age of 36 years, these patients were much younger than ours..$^{15}$ Another important difference between these mentioned studies and our series is the fact that we used patient self-assessment as the primary outcome measurement. Such an assessment was also used in another Dutch study that reviewed the results of 40 patients who underwent secondary submuscular trans- 
TABLE 2. Comparison of characteristics and clinical data between patients with an excellent/good outcome and those with a fair/poor outcome

\begin{tabular}{lccc}
\hline & \multicolumn{2}{c}{ Outcome } & \\
\cline { 2 - 3 } \multicolumn{1}{c}{ Characteristic } & $\begin{array}{c}\text { Excellent/ } \\
\text { Good }(n=16)\end{array}$ & $\begin{array}{c}\text { Fair/Poor } \\
(n=10)\end{array}$ & p Value \\
\hline Age (yrs) & $51(22-79)$ & $62(53-72)$ & $0.042^{*}$ \\
\hline Sex, F & $6(37.5)$ & $3(30)$ & 0.999 \\
\hline $\begin{array}{l}\text { Duration of symptoms } \\
\text { before 1st op (mos) }\end{array}$ & $10.0(2-18)$ & $14.5(4-60)$ & 0.186 \\
\hline Time btwn both ops (mos) & $10.5(5-22)$ & $12.5(5-26)$ & 0.698 \\
\hline Op on dominant hand & $8(50)$ & $6(60)$ & 0.701 \\
\hline Aspect of nerve, abnormal & $8(50)$ & $3(30)$ & 0.277 \\
\hline Symptoms before 1st op & & & \\
\hline \multicolumn{1}{c}{ Pain in 4th \& 5th digits } & $7 / 14(50)$ & $3 / 10(30)$ & 0.421 \\
\hline Tingling & $12 / 14(85.7)$ & $8 / 10(80)$ & 0.999 \\
\hline Numbness & $7 / 14(50)$ & $4 / 10(40)$ & 0.697 \\
\hline Weakness & $8 / 14(57.1)$ & $4 / 10(40)$ & 0.680 \\
\hline
\end{tabular}

Values are number (\%) of patients or median (range).

* Statistically significant.

position after a variety of primary procedures. ${ }^{2}$ Similar to our results, the discrepancy between clinical assessment and patient self-assessment in this study was remarkable; 8 patients had an excellent result according to the clinical assessment, but only 1 patient subjectively reported to be free of symptoms. Also in line with our findings, 3 of the 7 patients who reported worsening after surgery would, in hindsight, opt for surgery again. We agree with the authors that the assessment of outcome by the patient is more reliable than clinical assessment. This discrepancy between clinician-based and patient-based assessments, in which clinician-based results seem to be better than the opinion of the patients, partly explains differences in outcomes between our study and those in the literature. We found 1 other study that included patient-based assessments retrospectively and evaluated the results of revision surgery in 18 patients who were treated with secondary submuscular transposition. ${ }^{18}$ These authors found a satisfaction rate of $78 \%$. Moreover, $89 \%$ of the patients said that they would choose revision surgery again if they had to make the choice. However, again, patients in that study were younger than our patients (median 44 vs 56 years, respectively). Furthermore, their series included several patients with an extremely long interval between the first procedure and the revision surgery (up to 480 months), which makes comparison of the results with those of our series more difficult. Finally, another important difference between our study and most of the above-mentioned studies is the applied surgical technique (submuscular versus subcutaneous transposition in our study).

\section{Surgical Technique}

Theoretically, part of the modest results of ASTAFNUE could be explained by persisting compression of the ulnar nerve inherent to the applied subcutaneous technique. Alternatively, submuscular transposition can be performed
TABLE 3. Reported symptoms of all 26 patients after ASTAFNUE

\begin{tabular}{lccc}
\hline \multicolumn{1}{c}{ Symptom } & Improved (n [\%]) & Unchanged (n [\%]) & Worsened (n [\%]) \\
\hline Pain/tingling & $9(35)$ & $14(54)$ & $3(12)$ \\
\hline Weakness & $6(23)$ & $13(50)$ & $7(27)$ \\
\hline Numbness & $5(19)$ & $13(50)$ & $8(31)$ \\
\hline
\end{tabular}

Patients reported symptoms as improved, unchanged, or worsened for each clinical modality.

after FNUE. The supporters of this technique claim that the straight course of a submuscularly transposed nerve is better than when the nerve is transposed subcutaneously. An important detail in the technique we apply is that we always cut the medial intermuscular septum and open the antebrachial fascia to the medial epicondyle. By doing so, new compression after transposition is not only avoided, but the course of the ulnar nerve becomes natural. We do not feel that burying the ulnar nerve deep under the muscles contributes substantially to optimization of the course of the nerve. In addition, it is claimed that a submuscular position provides a better-protected, padded position. Although not systematically documented, patients have not expressed complaints that might be related to a vulnerable position of the subcutaneously located ulnar nerve. As far as we know, only 1 study performed a direct comparison between submuscular and subcutaneous transpositions. ${ }^{19}$ The applied subcutaneous transfer technique differs from ours in that both the intermuscular septum and the fascia were not addressed. We prefer subcutaneous transposition. It requires 48 hours of postoperative immobilization, whereas submuscular transposition requires 3 weeks of immobilization. Our preferred technique is a far less invasive procedure with which a sliding nerve environment is created. During revision surgery for patients with persisting complaints after previous submuscular transpositions, we have encountered massive scar formation around a completely fixed ulnar nerve. In addition, the total recovery period of subcutaneous transposition is much shorter than that after a submuscular procedure.

Rogers et al. ${ }^{15}$ found causes of continued pain after initial surgery to include retention of the medial intermuscular septum, dense perineural fibrosis of the nerve after intramuscular and subcutaneous transposition, adhesions of the nerve to the medial epicondylectomy site, and recurrent subluxation of the nerve over the medial epicondyle after subcutaneous transposition. In $92 \%$ of our patients, the epicondyle-olecranal ligament appeared to be (partially) intact during ASTAFNUE, which could be a result of secondary fibrosis or incomplete dissection in the primary surgery, as described by others in $1978 .{ }^{5}$ Another hypothesis for failure of primary and/or secondary surgery is irreversible damage of the ulnar nerve before surgery; however, this hypothesis is not supported by our findings. In our study, a normal appearance of the ulnar nerve during the transposition was not related to outcome.

\section{Timing of Surgery}

In general, prolonged nerve compression can cause ir- 
reversible axonal damage. For patients with UNE, usually 3 months of conservative treatment to wait for spontaneous recovery is advocated currently. It is not known what percentage of patients who initially present with UNE will indeed recover after conservative treatment. For those who do not, earlier timing of surgery might have led to a better outcome of neurolysis, should earlier identification have been possible. The same reasoning accounts for patients after ASTAFNUE. It is not known how long a patient should wait after neurolysis for UNE to expect any recovery. It might be that if symptoms do not alleviate within weeks after neurolysis, transposition should be performed at that time. At present, the interval between neurolysis and transposition is usually months, during which the ulnar nerve might still be compressed. Naturally, this compression is detrimental to the ulnar nerve and presumably affects the outcome of transposition negatively.

\section{Study Strengths and Limitations}

All the patients in our study had FNUE. ASTAFNUE was performed by an experienced nerve surgeon using the same technique in every patient. Because of this homogeneity, it is unlikely that differences in outcomes were the result of differences in treatment. In addition, we used a consecutive series of patients; therefore, it is unlikely that selection bias influenced the results in our study.

In addition to their older age, our patients might have been more difficult to treat than the patients in other studies because of the tertiary function of our center. However, it is difficult to compare the severity of the UNE of the patients at baseline because of differences in the way the degree of symptoms has been reported in the literature.

Recall bias might have played a part in our findings, as in every retrospective study. It was not possible to perform a new physical examination for practical reasons, because most patients lived relatively far away. We therefore evaluated current complaints via telephonic interview, performed systematically by a single, independent investigator.

\section{Conclusions}

Symptoms that persist after FNUE are difficult to treat. A majority of the patients in our series were satisfied after ASTAFNUE, but these satisfied patients included those whose initial symptoms resolved only partly or even deteriorated. Our modest outcomes show that ASTAFNUE does not resolve all symptoms, although the reasons are unknown. Apart from older age, no other predictors for an unsuccessful outcome were identified. In some of our patients, the ulnar nerve might have been intrinsically damaged from a substantial or prolonged entrapment that resulted in partial neurotmesis. Should this indeed have been the case, some of the patients might have been untreatable or should have undergone surgery sooner after the onset of complaints. Technical developments in nerve ultrasound ${ }^{4}$ or MRI ${ }^{16}$ might be helpful in further selecting patients for surgery, but studies on this subject have yet to be done. The modest results of ASTAFNUE should be mentioned when counseling patients with FNUE to manage their expectations. Patients, especially those who are elderly, should also consider the option of not undergoing a secondary procedure. A randomized trial that includes a conservative treatment group and groups of patients undergoing one of the several possible surgical procedures is needed to find the definitive answer for this clinical problem.

\section{References}

1. Aleem AW, Krogue JD, Calfee RP: Outcomes of revision surgery for cubital tunnel syndrome. J Hand Surg Am 39:2141-2149, 2014

2. Bartels RH, Grotenhuis JA: Anterior submuscular transposition of the ulnar nerve. For post-operative focal neuropathy at the elbow. J Bone Joint Surg Br 86:998-1001, 2004

3. Bartels RH, Verhagen WI, van der Wilt GJ, Meulstee J, van Rossum LG, Grotenhuis JA: Prospective randomized controlled study comparing simple decompression versus anterior subcutaneous transposition for idiopathic neuropathy of the ulnar nerve at the elbow: Part 1. Neurosurgery 56:522-530, 2005

4. Beekman R, Zijlstra W, Visser LH: A novel points system to predict the prognosis of ulnar neuropathy at the elbow. Muscle Nerve [epub ahead of print], 2016

5. Broudy AS, Leffert RD, Smith RJ: Technical problems with ulnar nerve transposition at the elbow: findings and results of reoperation. J Hand Surg Am 3:85-89, 1978

6. Caliandro P, La Torre G, Padua R, Giannini F, Padua L: Treatment for ulnar neuropathy at the elbow. Cochrane Database Syst Rev 7:CD006839, 2012

7. Caputo AE, Watson HK: Subcutaneous anterior transposition of the ulnar nerve for failed decompression of cubital tunnel syndrome. J Hand Surg Am 25:544-551, 2000

8. Chen HW, Ou S, Liu GD, Fei J, Zhao GS, Wu LJ, et al: Clinical efficacy of simple decompression versus anterior transposition of the ulnar nerve for the treatment of cubital tunnel syndrome: A meta-analysis. Clin Neurol Neurosurg 126:150-155, 2014

9. Gabel GT, Amadio PC: Reoperation for failed decompression of the ulnar nerve in the region of the elbow. J Bone Joint Surg Am 72:213-219, 1990

10. Gervasio O, Gambardella G, Zaccone C, Branca D: Simple decompression versus anterior submuscular transposition of the ulnar nerve in severe cubital tunnel syndrome: a prospective randomized study. Neurosurgery 56:108-117, 2005

11. Goldfarb CA, Sutter MM, Martens EJ, Manske PR: Incidence of re-operation and subjective outcome following in situ decompression of the ulnar nerve at the cubital tunnel. J Hand Surg Eur Vol 34:379-383, 2009

12. Keiner D, Gaab MR, Schroeder HW, Oertel J: Comparison of the long-term results of anterior transposition of the ulnar nerve or simple decompression in the treatment of cubital tunnel syndrome-a prospective study. Acta Neurochir (Wien) 151:311-316, 2009

13. Krogue JD, Aleem AW, Osei DA, Goldfarb CA, Calfee RP: Predictors of surgical revision after in situ decompression of the ulnar nerve. J Shoulder Elbow Surg 24:634-639, 2015

14. Novak CB, Mackinnon SE: Selection of operative procedures for cubital tunnel syndrome. Hand (NY) 4:50-54, 2009

15. Rogers MR, Bergfield TG, Aulicino PL: The failed ulnar nerve transposition. Etiology and treatment. Clin Orthop Relat Res (269): 193-200, 1991

16. Shen L, Masih S, Patel DB, Matcuk GR Jr: MR anatomy and pathology of the ulnar nerve involving the cubital tunnel and Guyon's canal. Clin Imaging 40:263-274, 2016

17. Soltani AM, Best MJ, Francis CS, Allan BJ, Panthaki ZJ: Trends in the surgical treatment of cubital tunnel syndrome: an analysis of the national survey of ambulatory surgery database. J Hand Surg Am 38:1551-1556, 2013

18. Vogel RB, Nossaman BC, Rayan GM: Revision anterior 
submuscular transposition of the ulnar nerve for failed subcutaneous transposition. Br J Plast Surg 57:311-316, 2004

19. Zarezadeh A, Shemshaki H, Nourbakhsh M, Etemadifar MR, Moeini M, Mazoochian F: Comparison of anterior subcutaneous and submuscular transposition of ulnar nerve in treatment of cubital tunnel syndrome: A prospective randomized trial. J Res Med Sci 17:745-749, 2012

20. Zlowodzki M, Chan S, Bhandari M, Kalliainen L, Schubert W: Anterior transposition compared with simple decompression for treatment of cubital tunnel syndrome. A meta-analysis of randomized, controlled trials. J Bone Joint Surg Am 89:2591-2598, 2007

\section{Disclosures}

The authors report no conflict of interest concerning the materi- als or methods used in this study or the findings specified in this paper.

\section{Author Contributions}

Conception and design: Datema, Eekhof, Malessy. Acquisition of data: van Gent, Eekhof. Analysis and interpretation of data: Datema, van Gent, Malessy. Drafting the article: Datema, van Gent. Critically revising the article: Groen, Pondaag, Malessy. Approved the final version of the manuscript on behalf of all authors: Datema. Statistical analysis: Datema, Groen. Study supervision: Malessy.

\section{Correspondence}

Mirjam Datema, Leiden University Medical Centre, Postbus 9600, 2300 RC Leiden, The Netherlands. email: m.datema@lumc.nl. 